\title{
Remote ischemic postconditioning protects the heart by upregulating ALDH2 expression levels through the PI3K/Akt signaling pathway
}

\author{
YING YU $^{1,2}$, XIAN-JIE JIA ${ }^{3}$, QIAO-FENG ZONG ${ }^{1}$, GUAN-JUN ZHANG ${ }^{1}$, HONG-WEI YE ${ }^{1}$, \\ JIE HU ${ }^{1}$, QIN GAO ${ }^{1,2}$ and SU-DONG GUAN ${ }^{1}$ \\ ${ }^{1}$ Department of Physiology; ${ }^{2}$ Anhui Key Laboratory of Tissue Transplantation; \\ ${ }^{3}$ Department of Epidemiology and Statistics, Bengbu Medical College, \\ Bengbu, Anhui 233030, P.R. China
}

Received September 28, 2013; Accepted March 18, 2014

DOI: $10.3892 / \mathrm{mmr} .2014 .2156$

\begin{abstract}
Remote ischemic postconditioning (RIPostC) has been demonstrated to protect the myocardium against ischemia/reperfusion (I/R) injury; however, the mediator and underlying mechanisms remain to be elucidated. It has been confirmed that aldehyde dehydrogenase 2 (ALDH2) is involved in the remote ischemic preconditioning pathway, but whether it is involved in RIPostC remains unknown. The aim of the present study was to determine whether increased ALDH2 expression levels were involved in the cardioprotective effect evoked by RIPostC via the phosphatidylinositol-3-kinase (PI3K)/Akt signaling pathway. Male Sprague Dawley rats $(n=48)$ were randomly allocated into the following four groups: Sham group, I/R group, RIPostC group, and RIPostC plus wortmannin group (RIPostC+Wort). With the exception of the Sham group, the anesthetized rats underwent $45 \mathrm{~min}$ of coronary artery occlusion followed by $180 \mathrm{~min}$ of reperfusion to mimic an $\mathrm{I} / \mathrm{R}$ injury model. Hemodynamic parameters, including the mean arterial pressure and heart rate, were recorded, the infarct size was determined and the plasma lactate dehydrogenase (LDH) content and creatine kinase (CK) activity levels were measured. The expression levels of Bcl-2 and Bax at the mRNA level and ALDH2, Akt, phospho-Akt (p-Akt), caspase-3 and cleaved caspase-3 at the protein level in the left anterior myocardium were assessed. In the RIPostC group, the infarct size was reduced versus that of the I/R group. The plasma $\mathrm{LDH}$ content and CK activity levels were also reduced. The expression levels of ALDH2 protein were elevated, accompanied with increases in the levels of Bcl-2/Bax and p-Akt/Akt and a reduction in
\end{abstract}

Correspondence to: Dr Qin Gao, Department of Physiology, Bengbu Medical College, 2600 Dong Hai Avenue, Bengbu, Anhui 233030, P.R. China

E-mail: bbmcgq@126.com

Key words: remote ischemic postconditioning, ALDH2, cardioprotection, ischemia/reperfusion, Akt the levels of cleaved caspase-3. When the PI3K inhibitor wortmannin was administered at reperfusion, the $\mathrm{p}$-Akt/Akt ratio was markedly reduced and associated with a reduction in the ALDH2 and Bcl-2/Bax levels, and the cleaved caspase-3 expression levels were elevated. In conclusion, ALDH2 may be an important mediator in the cardioprotection of RIPostC through the PI3K/Akt-dependent signaling pathway.

\section{Introduction}

Myocardial ischemia/reperfusion (I/R) injury is a phenomenon that is frequently evident in clinical practice (1). Although ischemic preconditioning (IPC) and remote ischemic preconditioning (RIPreC) significantly reduce myocardial injury $(2,3)$, the applicability of IPC is limited by the unpredictable nature of ischemic events in clinical practice. Ischemic postconditioning (IPostC) is triggered during the clinically applicable time period of reperfusion, with the major limitation being the invasive protocol (4).

Remote ischemic postconditioning (RIPostC) is another endogenous cardioprotective method (5). Brief application of ischemic stimulus on an organ at a distance from the heart may render the heart more tolerant to the subsequential prolonged period of ischemia. RIPostC does not require invasive intervention, unlike other types of intervention, and may have a promising future in clinical practice (6). Therefore, the present study concerning the mechanism by which RIPostC induces cardioprotection against I/R injury is beneficial for further studies and may help to identify a novel interventional method for use in clinical practice $(7,8)$.

Myocardial I/R injury leads to an overload of oxidative stress, an imbalance of calcium homeostasis and cell apoptosis, and thereby induces tissue damage. Mitochondria have a fundamental role in the maintenance of the normal structure and function of tissues. A decline in mitochondrial function has a critical role in the promotion of cell apoptosis. Aldehyde dehydrogenase 2 (ALDH2), a type of mitochondrial protein enzyme involved in the metabolism of acetaldehyde and other toxic aldehydes (9), is considered to be responsible for the oxidation and detoxification of reactive aldehydes in different 
organs and cell types $(10,11)$. ALDH2 is highly expressed in the heart, liver, kidney and muscles (12). Chen et al (13) indicated an inverse correlation between the levels of ALDH2 activity and infarct size in a myocardial infarction model. ALDH2 is the crucial enzyme for the removal of aldehydes in the heart, and activated ALDH2 significantly reduces cardiac I/R injury (14). A previous study has shown that the cardiac ALDH2 expression levels were further reduced with the development of diabetes. Thus, ALDH2 may be an endogenous cardiac protective factor in myocardial injury. Furthermore, it was also demonstrated in a previous study that RIPostC produced a protective effect through inhibition of the opening of the mitochondrial permeability transition pore (mPTP) and apoptosis (15). However, the role of ALDH2 in RIPostC cardioprotection has not been elucidated. The purpose of the present study was to investigate whether ALDH2 is involved in the protective role of RIPostC.

The reperfusion injury salvage kinase (RISK) signaling pathway refers to a group of prosurvival kinases, including the phosphatidylinositol-3 kinase (PI3K)/Akt cascade, which confer cardioprotection when specifically activated at the onset of myocardial reperfusion following ischemia (16). Furthermore, a previous study has established that activation of the PI3K/Akt signaling pathway contributes to IPostC-mediated cardioprotection (17). However, it remains unknown whether the PI3K/Akt signaling pathway is involved in the cardioprotection of RIPostC.

In the present study, the objectives were: i) To investigate the role of ALDH2 in RIPostC; ii) to determine whether RIPostC mediates its protective effect through the activation of the PI3K/Akt-dependent signaling pathway; and iii) to clarify the association of ALDH2 and the PI3K/Akt signaling pathway in the protective effect of RIPostC.

\section{Materials and methods}

Animals and materials. Male Sprague-Dawley rats (250-300 g) were obtained from the Animal Center of Bengbu Medical College (Bengbu, China). All rats were housed in individual cages in a temperature-controlled room with a 12-h light/dark cycle. The rats were fed a normal diet and had free access to distilled water. All the animal procedures were in accordance with the United States National Institutes of Health Guide and were approved by the Animal Use and Care Committee of Bengbu Medical College.

Chemicals and reagents. Wortmannin was purchased from Sigma-Aldrich (St. Louis, MO, USA). Lactate dehydrogenase (LDH) and creatine kinase (CK) assay kits were purchased from Nanjing Jiancheng Bioengineering Institute (Nanjing, China). All primers were purchased from Sangon Biotech Co., Ltd. (Shanghai, China). Mouse ALDH2 and $\beta$-actin antibodies were purchased from Santa Cruz Biotechnology, Inc. (Santa Cruz, CA, USA), and rabbit polyclonal Akt, phospho-Akt (p-Akt), caspase-3 and cleaved caspase-3 antibodies were purchased from Anbo Biotechnology Co., Ltd (San Francisco, CA, USA). A chemiluminescence reaction (ECL) system was purchased from Millipore (Billerica, MA, USA).

Animal experiment. Male Sprague Dawley rats $(n=48)$ were randomly divided into the following four groups $(n=12$ in each group): Sham group, I/R group (I/R), RIPostC group (RIPostC), and RIPostC + wortmannin group (RIPostC+Wort). After the rats were anesthetized with $60 \mathrm{mg} / \mathrm{kg}$ pentobarbital sodium through intraperitoneal injection, the left anterior descending coronary arteries (LAD) of all rats were encircled with a suture to make a snare after their chests had been opened. With the exception of the Sham group, the LAD were ligated for $45 \mathrm{~min}$ (ischemia) followed by $180 \mathrm{~min}$ of the LAD open (reperfusion) in vivo. In the RIPostC group, three cycles of lower limb I/R (right femoral artery clamping for $5 \mathrm{~min}$ and declamping for $5 \mathrm{~min}$ ) were performed prior to the onset of myocardial reperfusion (6). In the $\mathrm{RIPostC}+$ Wort group, wortmannin (15 $\mu \mathrm{g} / \mathrm{kg}$; a PI3K inhibitor) was administered intravenously $30 \mathrm{sec}$ prior to myocardial reperfusion in RIPostC-treated animals. Throughout the whole process of the experiment, the mean arterial pressure (MAP) and heart rate (HR) were continuously monitored.

Assessment of myocardial infarct size. The infarct size was measured in all groups at the end of the reperfusion. The heart was removed with the reoccluded LAD, and was retrogradely perfused with $1.5 \mathrm{ml} 1 \%$ Evans blue dye to delineate the risk area. Following freezing at $-20^{\circ} \mathrm{C}$, the heart was cut into 5-6 sections from the apex to the base and incubated in $1 \%$ triphenyltetrazolium chloride for $15 \mathrm{~min}$. Subsequently, the sections were fixed in $10 \%$ formalin buffer, and the infarct size was quantified by computerized planimetry using ImageJ software (version 1.40, National Institutes of Health, Bethesda, MD, USA). The myocardial infarct size was expressed as the percentage of the area at risk.

Measurement of LDH content and CK activity levels in the plasma. At the end of the reperfusion, an arterial blood sample was placed in test tubes with heparin and centrifuged at $1509 \mathrm{x} \mathrm{g}$ for $30 \mathrm{~min}$. The supernatant was collected and stored at $-20^{\circ} \mathrm{C}$, thawed once and assayed. The LDH content and $\mathrm{CK}$ activity levels were measured spectrophotometrically at wavelengths of 440 and $660 \mathrm{~nm}$ using colorimetric assay kits according to the manufacturer's instructions $(18,19)$.

Reverse transcription polymerase chain reaction (RT-PCR) assay for determining the B-cell lymphoma 2 (Bcl-2) and Bcl-2-associated X protein (Bax) mRNA levels. Total RNA was extracted from the left anterior myocardium using TRIzol reagent (Life Technologies Corporation, Carlsbad, CA, USA) according to the manufacturer's instructions. The total RNA $(2 \mu \mathrm{g})$ was reversely transcribed to cDNA, and PCR was performed by a routine method. The sequences of the primers for Bax, Bcl-2 and $\beta$-actin are shown in Table I. The PCR products were analyzed on $1 \%$ agarose gel. The densitometry results for the Bcl-2 and Bax genes were compared with the corresponding $\beta$-actin levels to account for loading differences.

Western blot analysis of ALDH2, Akt and p-Akt, caspase-3 and cleaved caspase-3. Left anterior myocardium tissues from each group were collected and homogenized in a lysis buffer, which contained $20 \mathrm{mmol} / \mathrm{l}$ Tris ( $\mathrm{pH} 7.4$ ), $150 \mathrm{mmol} / \mathrm{l} \mathrm{NaCl}$, $1 \mathrm{mmol} / 1$ EDTA, $1 \mathrm{mmol} / 1$ EGTA, $1 \%$ Triton, $0.1 \%$ SDS, and $1 \%$ protease inhibitor cocktail. The homogenates were sonicated and centrifuged at $12,000 \mathrm{x}$ g for $30 \mathrm{~min}$ at $4^{\circ} \mathrm{C}$. The 
Table I. Quantitative polymerase chain reaction primers for Bax, Bcl-2 and $\beta$-actin.

\begin{tabular}{llll}
\hline Gene & Primer & Sequence & Product (bp) \\
\hline Bax & Forward & 5'-GGA TCG AGC AGA GAG GAT GG-3' \\
& Reverse & 5'-TGG TGA GTG AGG CAG TGA GG-3' \\
Bcl-2 & Forward & 5'-CTG GTG GAC AAC ATC GCT CTG-3' \\
& Reverse & 5'-GGT CTG CTG ACC TCA CTT GTG-3' & 464 \\
$\beta$-actin & Forward & 5'-GAT GGT GGG TAT GGG TCA GAA GGA C-3' & 227 \\
& Reverse & 5'-GCT CAT TGC CGA TAG TGA TGA CT-3' & 630 \\
\hline
\end{tabular}

Bcl-2, B-cell lymphoma 2; Bax, Bcl-2-associated X protein.

Table II. Hemodynamic data in the rats.

\begin{tabular}{|c|c|c|c|c|c|}
\hline \multirow[b]{2}{*}{ Group } & \multirow[b]{2}{*}{ Baseline } & \multirow{2}{*}{$\frac{\text { Ischemia (min) }}{45}$} & \multicolumn{3}{|c|}{ Reperfusion (min) } \\
\hline & & & 10 & 60 & 180 \\
\hline \multicolumn{6}{|l|}{ MAP (mmHg) } \\
\hline Sham & $109.58 \pm 3.10$ & $111.33 \pm 7.95$ & $103.33 \pm 3.02$ & $102.50 \pm 3.32$ & $97.83 \pm 3.73$ \\
\hline $\mathrm{I} / \mathrm{R}$ & $108.42 \pm 2.84$ & $82.92 \pm 2.76^{\mathrm{a}}$ & $63.83 \pm 3.83^{\mathrm{a}}$ & $80.92 \pm 3.82^{\mathrm{a}}$ & $72.42 \pm 4.05^{\mathrm{a}}$ \\
\hline RIPostC & $111.58 \pm 2.66$ & $84.42 \pm 3.96^{\mathrm{a}}$ & $74.42 \pm 2.56^{\mathrm{a}}$ & $81.00 \pm 3.63^{\mathrm{a}}$ & $71.42 \pm 4.77^{\mathrm{a}}$ \\
\hline RIPostC+Wort & $104.42 \pm 3.66$ & $77.50 \pm 3.78^{a}$ & $65.42 \pm 2.40^{\mathrm{a}}$ & $78.00 \pm 3.04^{\mathrm{a}}$ & $72.42 \pm 3.70^{\mathrm{a}}$ \\
\hline \multicolumn{6}{|l|}{ HR (beats/min) } \\
\hline Sham & $413.58 \pm 9.33$ & $399.25 \pm 4.42$ & $395.50 \pm 3.87$ & $384.00 \pm 7.35$ & $386.92 \pm 3.19$ \\
\hline $\mathrm{I} / \mathrm{R}$ & $412.33 \pm 7.67$ & $354.75 \pm 10.65^{a}$ & $322.00 \pm 8.49^{\mathrm{a}}$ & $340.83 \pm 7.85^{\mathrm{a}}$ & $318.50 \pm 7.51^{\mathrm{a}}$ \\
\hline RIPostC & $413.33 \pm 8.47$ & $369.00 \pm 9.09^{\mathrm{a}}$ & $341.17 \pm 5.88^{a}$ & $371.92 \pm 11.58$ & $364.08 \pm 8.27$ \\
\hline RIPostC+Wort & $403.08 \pm 7.91$ & $353.58 \pm 11.05^{\mathrm{a}}$ & $323.67 \pm 8.53^{\mathrm{a}}$ & $344.67 \pm 8.07^{\mathrm{a}}$ & $309.75 \pm 8.73^{\mathrm{a}}$ \\
\hline
\end{tabular}

Values are the mean \pm standard error $(n=12) .{ }^{a} \mathrm{P}<0.01$ vs. baseline. Sham, LAD with no other intervention; I/R, ischemia/reperfusion; RIPostC, remote ischemic post conditioning; Wort, wortmanin $(15 \mu \mathrm{g} / \mathrm{kg}$; a PI3K inhibitor); MAP, mean arterial pressure; HR, heart rate.

protein concentration was determined using a bicinchoninic acid protein assay kit (Beyotime Institute of Biotechnology, Shanghai, China). The total protein $(80 \mu \mathrm{g})$ was separated by SDS-PAGE, and transferred electrophoretically to polyvinylidene difluoride filter membranes (14).

The membranes were blocked with $5 \%$ nonfat milk in Tris-buffered saline with Tween 20 for $2 \mathrm{~h}$, and then they were incubated at $4^{\circ} \mathrm{C}$ overnight with the corresponding primary antibody, including mouse ALDH2 antibody (1:500), $\beta$-actin antibody (1:500), rabbit Akt antibody $(1: 1,000)$, rabbit p-Akt antibody $(1: 1,000)$, rabbit caspase-3 antibody $(1: 1,000)$ and rabbit cleaved caspase-3 antibody $(1: 1,500)$. All membranes were incubated for $1 \mathrm{~h}$ with the corresponding horseradish peroxidase (HRP)-linked anti-mouse immunoglobulin ( $\operatorname{IgG}$ ) or HRP-linked anti-rabbit IgG secondary antibody. The membranes were analyzed by an ECL system. The autoradiographs were scanned and the band densities were determined with Image J software.

Statistical analysis. Data are expressed as the mean \pm standard error of the mean. One-way analysis of variance followed by Student-Newman-Keuls was used for multiple comparisons. Differences with $\mathrm{P}<0.05$ were considered statistically significant. All data were analyzed using GraphPad Prism version 4.0 software (GraphPad Software, Inc., San Diego, CA, USA).

\section{Results}

Hemodynamics. With the exception of the Sham group, the MAP and HR were reduced in each group following the coronary artery occlusion, and recovered to varying extents after $180 \mathrm{~min}$ of reperfusion. No statistical differences in the MAP and HR among these groups were identified (Table II).

Myocardial infarct size and lactate dehydrogenase and creatine kinase levels in the plasma. The myocardial infarct size (percentage infarct size/area at risk) was reduced by $\geq 55 \%$ in the RIPostC group $(31.25 \pm 1.89 \%)$ compared with that in the I/R group $(55.75 \pm 2.21 \%$; $\mathrm{P}<0.01)$. However, wortmannin $(15 \mu \mathrm{g} / \mathrm{kg})$, an inhibitor of the PI3K/Akt signaling pathway, attenuated the effect of RIPostC (RIPostC+Wort, $49.67 \pm 3.48$ vs. RIPostC, $31.25 \pm 1.89 \%, \mathrm{P}<0.01$; Fig. 1).

As displayed in Fig. 2, compared with those in the I/R group, the LDH content (RIPostC, 324.25 12.46 vs. I/R, $394.08 \pm 10.83 \mathrm{U} / 1, \mathrm{P}<0.01$ ) and $\mathrm{CK}$ activity levels (RIPostC, 


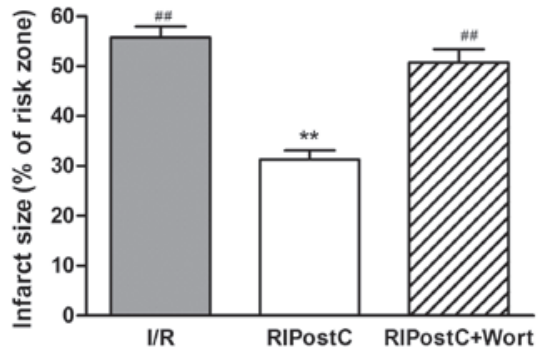

Figure 1. Effects of RIPostC and Wort on infarct size in the rat myocardium in vivo. Values are the mean \pm standard error $(\mathrm{n}=4) .{ }^{* *} \mathrm{P}<0.01$ vs. $\mathrm{I} / \mathrm{R} ;{ }^{\# \prime} \mathrm{P}<0.01$ vs. RIPostC. I/R, ischemia/reperfusion; RIPostC, remote ischemic postconditioning; Wort, wortmannin.

A

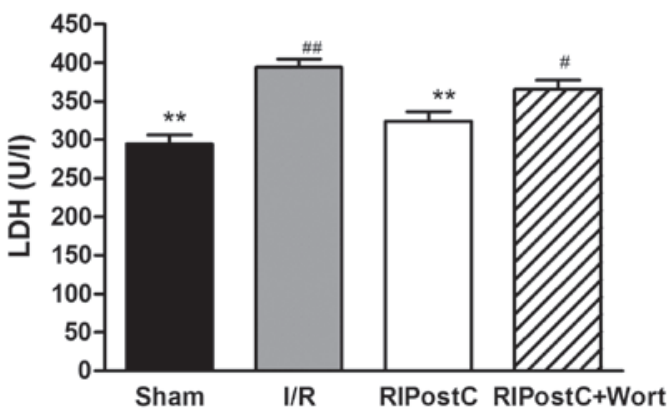

B

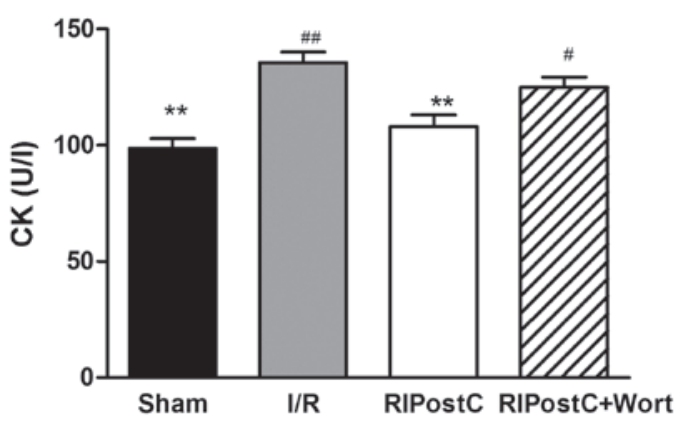

Figure 2. Effects of RIPostC and Wort on the plasma of (A) LDH content and (B) CK activity in rats. Values are the mean \pm standard error $(n=12) .{ }^{* *} \mathrm{P}<0.01$ vs. I/R; ${ }^{\prime} \mathrm{P}<0.05,{ }^{\# \#} \mathrm{P}<0.01$ vs. RIPostC. I/R, ischemia/reperfusion; RIPostC, remote ischemic postconditioning; Wort, wortmannin; LDH, lactate dehydrogenase; CK, creatine kinase.

$108.08 \pm 4.96$ vs. I/R, 135.58 $\pm 4.61 \mathrm{U} / 1, \mathrm{P}<0.01)$ were significantly reduced in the RIPostC group. However, in contrast to those of the RIPostC group, the LDH content (RIPostC+Wort, $365.92 \pm 11.51$ vs. RIPostC, 324.25 $\pm 12.46 \mathrm{U} / 1, \mathrm{n}=12, \mathrm{P}<0.05$ ) and CK activity levels (RIPostC+Wort, $125.00 \pm 4.48$ vs. RIPostC, $108.08 \pm 4.96 \mathrm{U} / 1, \mathrm{n}=12, \mathrm{P}<0.05)$ were markedly increased in the RIPostC+Wort group.

Changes in the expression levels of myocardial Bcl-2 and Bax at the mRNA level and cleaved caspase-3 and caspase-3 at the protein level. The RT-PCR results revealed that, compared with those in the I/R group, the Bcl-2/Bax ratio at the mRNA level was elevated in the Sham and RIPostC groups (Sham, $1.43 \pm 0.05$; RIPostC, $1.32 \pm 0.06$ vs. I/R, 0.31 $\pm 0.03, \mathrm{n}=4, \mathrm{P}<0.01$; Fig. 3). However, compared with that in the RIPostC group, the ratio was significantly reduced in the RIPostC+Wort group $(0.43 \pm 0.04)$.

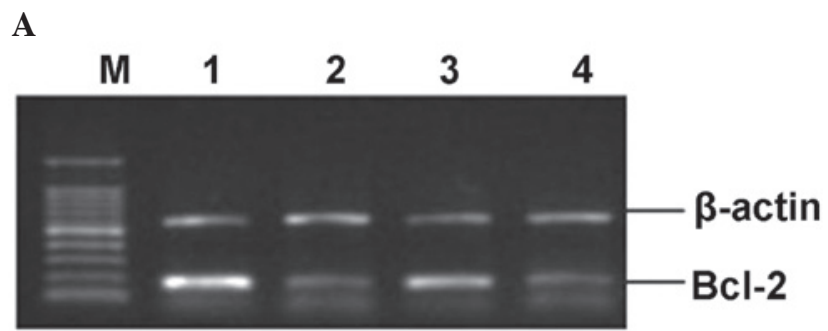

B

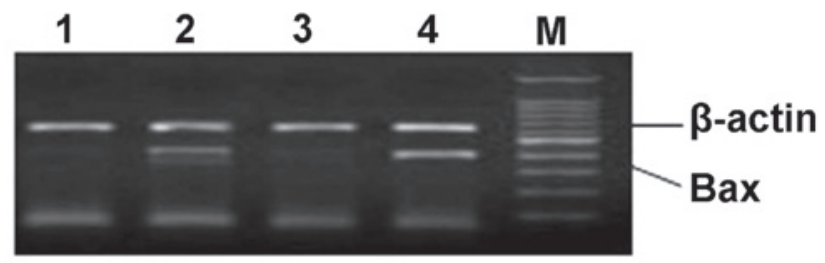

C

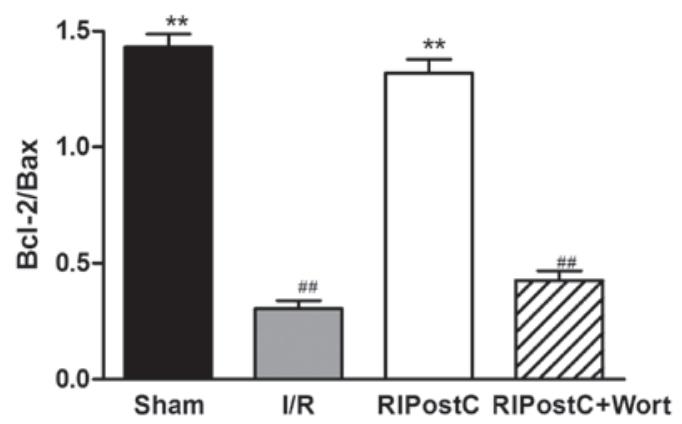

Figure 3. Expression levels of myocardial (A) Bcl-2 and (B) Bax mRNA and (C) quantification of the Bcl-2/Bax ratio for the different groups. Values are the mean \pm standard error $(n=4)$. ${ }^{* *} \mathrm{P}<0.01$ vs. I/R; ${ }^{\# \#} \mathrm{P}<0.01$ vs. RIPostC. $\mathrm{M}$, marker; 1, Sham; 2, I/R; 3, RIPostC; 4, RIPostC+Wort. I/R, ischemia/reperfusion; RIPostC, remote ischemic postconditioning; Wort, wortmannin; Bcl-2, B-cell lymphoma 2; Bax, Bcl-2-associated X protein.

As shown in Fig. 4, only small amounts of cleaved caspase-3 were detected in the left anterior myocardium in the Sham group. In contrast to those of the Sham group, the expression levels of cleaved caspase- 3 and the ratio of cleaved caspase-3/caspase-3 were increased in the I/R group (I/R, $0.64 \pm 0.03$ vs. Sham, $0.20 \pm 0.02, \mathrm{n}=4, \mathrm{P}<0.01)$. Compared with those in the I/R group, the levels of cleaved caspase-3 were reduced and the ratio of cleaved caspase-3/caspase- 3 was suppressed in the RIPostC group (RIPostC, $0.31 \pm 0.04$ vs. I/R, 0.64 $\pm 0.03, \mathrm{P}<0.01)$. However, treatment with wortmannin significantly attenuated the effect of RIPostC $(0.72 \pm 0.07)$.

Changes in the myocardial ALDH2, Akt and p-Akt protein levels. The western blot analysis revealed that, compared with that of the I/R group, the ratio of ALDH $2 / \beta$-actin at the protein level in the RIPostC group was increased (RIPostC, $0.83 \pm 0.03$ vs. I/R, 0.41 $\pm 0.03, \mathrm{n}=4, \mathrm{P}<0.01)$. Compared with that of the RIPostC group, the ratio in the RIPostC+Wort group was reduced (0.49 \pm 0.03 ; Fig. 5).

Also in the western blot analysis, compared with that of the I/R group, the ratio of $\mathrm{p}-\mathrm{Akt} / \mathrm{Akt}$ in the RIPostC group was increased (RIPostC: $0.74 \pm 0.04$ vs. I/R: $0.26 \pm 0.04, n=4, P<0.01$ ), and compared with that of the RIPostC group, the ratio in the RIPostC+Wort group was reduced (0.31 \pm 0.04 ; Fig. 6). 
A

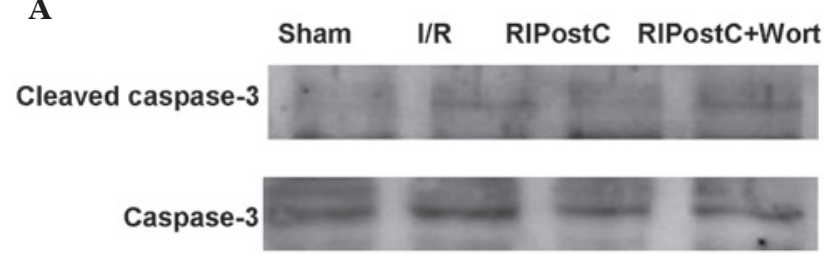

B

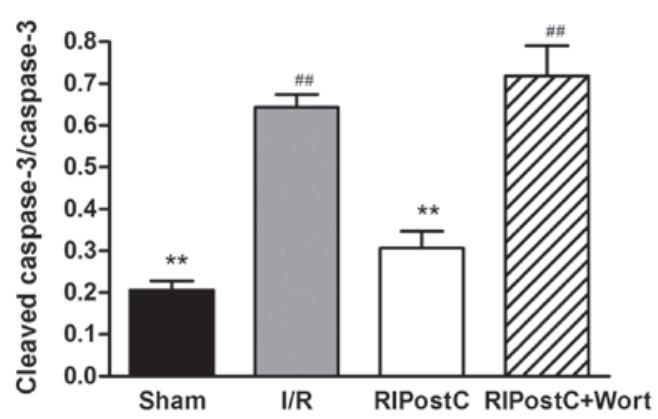

Figure 4. (A) Representative western blot and (B) quantitative analysis of the cleaved caspase-3/caspase-3 protein expression ratios in the myocardium of the rats treated with RIPostC and Wort. Values are the mean \pm standard error $(\mathrm{n}=4) .{ }^{* *} \mathrm{P}<0.01$ vs. I/R; ${ }^{\#} \mathrm{P}<0.01$ vs. RIPostC. I/R, ischemia/reperfusion; RIPostC, remote ischemic postconditioning; Wort, wortmannin.

\section{Discussion}

Studies have demonstrated that ALDH2 is a mitochondrial enzyme involved in protecting the heart against I/R injury $(20,21)$. In the present study, the effect of RIPostC was evaluated and the role of ALDH2 in the RIPostC cardioprotective effect on myocardial I/R injury was observed. As the results showed, the expression levels of ALDH2 were reduced following reperfusion in the I/R model compared with those in the Sham group. The findings of the present study also demonstrated that RIPostC reduced the myocardial infarct size, the levels of plasma LDH and CK release, and the levels of cleaved caspase- 3 protein, while the expression levels of $\mathrm{ALDH} 2$ protein, and the $\mathrm{p}-\mathrm{Akt} / \mathrm{Akt}$ and $\mathrm{Bcl}-2 / \mathrm{Bax}$ ratio levels were increased in contrast to those of the $\mathrm{I} / \mathrm{R}$ group. All the results suggested that ALDH2 may participate in the RIPostC cardioprotective effect and activation of ALDH2 expression may reduce the levels of cardiomyocyte apoptosis. The present study further identified that when the RIPostC rats were treated with wortmannin, a PI3K inhibitor, the protective effect was abrogated, the p-Akt/Akt ratio was markedly reduced, and was associated with decreases in the ALDH2 and Bcl-2/Bax levels, and the cleaved caspase-3 expression levels were increased. All these findings indicated that RIPostC had a cardioprotective and anti-apoptotic effect through activation of the PI3K/Akt-dependent signaling pathway, and upregulation of the ALDH2 expression levels; thus, ALDH2 may be an important mediator of RIPostC.

RIPostC, which had been demonstrated to confer cardioprotection, was first reported by Kerendi et al in 2005 (5). RIPostC was later reported to have a cardioprotective effect in various animal models, including rabbits, pigs and rats (22-24). Furthermore, an increasing number of clinical studies investigated the possible protection by remote ischemic-conditioning, mostly in cardiac and major vascular
A Sham I/R RIPostC RIPostC+Wort

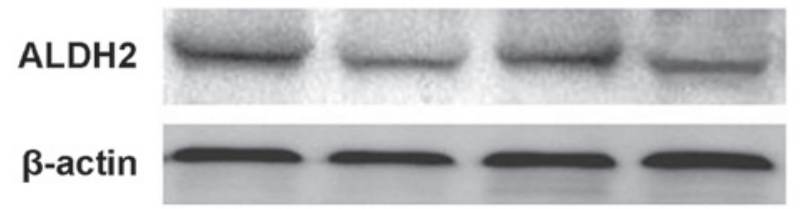

B

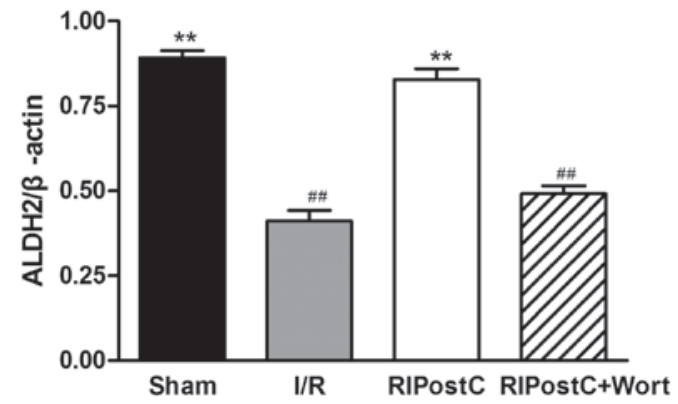

Figure 5. (A) Representative western blot and (B) quantitative analysis of the ALDH2/ $\beta$-actin protein expression ratios in the myocardium of the rats treated with RIPostC and Wort. Values are the mean \pm standard error $(n=4)$. ${ }^{* * *} \mathrm{P}<0.01$ vs. I/R; ${ }^{\# \#} \mathrm{P}<0.01$ vs. RIPostC. I/R, ischemia/reperfusion; RIPostC, remote ischemic postconditioning; Wort, wortmannin; ALDH2, aldehyde dehydrogenase 2 .

A Sham I/R RIPostC RIPostC+Wort p-Akt

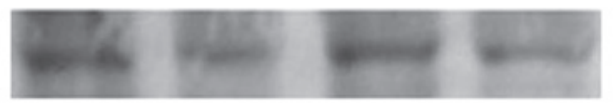

Akt

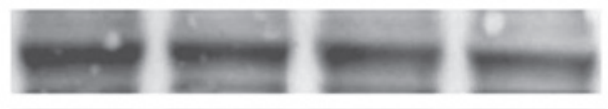

$\beta$-actin

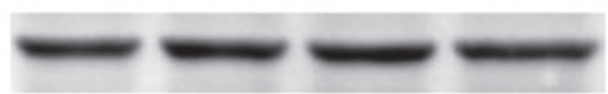

$\mathbf{B}$

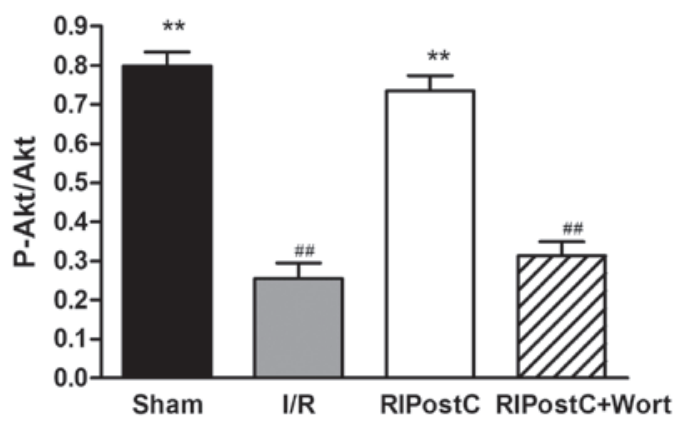

Figure 6. (A) Representative western blot and (B) quantitative analysis of the p-Akt/Akt protein expression ratios in the myocardium of the rats treated with RIPostC and Wort. Values are the mean \pm standard error $(n=4) .{ }^{* * *} \mathrm{P}<0.01$ vs. I/R; ${ }^{\# \# ~} \mathrm{P}<0.01$ vs. RIPostC. I/R, ischemia/reperfusion; RIPostC, remote ischemic postconditioning; Wort, wortmannin.

surgery $(25,26)$. Remote ischemic-conditioning, which consists of transient limb ischemia applied prior to or during a prolonged period of ischemia, improved the outcome of patients suffering from acute ischemia or who were at risk of developing ischemia during surgery (27). Although the protective role has been widely recognized, the underlying mechanisms remain to be fully elucidated. The objective of the present study was to confirm whether cardiac function is 
protected through RIPostC by an increase in the expression levels of ALDH2 and a reduction in the levels of cardiomyocyte apoptosis.

ALDH2 is a mitochondrial enzyme which is abundantly expressed in the heart. ALDH2 has a key role in the metabolism of acetaldehyde and other toxic aldehydes to confer cardioprotection (28). However, excessive levels of aldehydes severely damage cardiomyocytes and cardiac function. Therefore, the myocardial infarction and the levels of LDH and CK are frequently used to quantify the amount of myocardial damage. The findings of the present study showed that RIPostC reduced the myocardial infarct size and inhibited the release of $\mathrm{LDH}$ and $\mathrm{CK}$, while increasing the levels of ALDH2 protein compared with those in the I/R group. RIPostC may have protected the heart by upregulating the ALDH2 expression levels against the I/R injury in the rat model in vivo.

Numerous studies $(13,29)$ have indicated that ALDH2 has a key role in the cardioprotection against I/R injury, as the overexpression of ALDH2 improves cardiac function and reduces the levels of cardiomyocyte apoptosis. Prior to the present study, it had already been reported that damage of the myocardial ultrastructure accompanied the aggravation of oxidative stress in diabetic rats. However, when ethanol was administered to the diabetic rats in order to induce ALDH2 activity, the events of oxidative stress, the destruction of the myocardial function and the occurrence of apoptosis were attenuated (14,30). Those findings suggested that a ALDH2 deficiency aggravated cardiac dysfunction, while ALDH2 activation protected the heart against myocardial injury, possibly through detoxification of toxic aldehyde. Evidence from previous studies also revealed that ethanol postconditioning had a cardioprotective effect by upregulating the levels of ALDH 2 mRNA expression and inhibiting the opening of the MPTP (31), which is considered to be an important factor in apoptosis. In regard to RIPostC cardioprotection, the present study aimed to observe whether $\mathrm{ALDH} 2$ reduced the levels of apoptosis.

Cardiomyocyte apoptosis is one of the major pathogenic mechanisms underlying myocardial I/R injury (32). Bcl-2 family proteins are potent regulators of the mitochondrial changes during apoptosis. The balance in the expression levels of the antiapoptotic Bcl-2 and the proapoptotic Bax proteins has a major role in the regulation of myocardial apoptotic cell death. The Bcl-2/Bax ratio represents the extent of apoptosis $(33,34)$. Caspases are regarded as the central executors of the apoptotic pathway. Among the family of caspases, caspase-3 ultimately executes the apoptotic signal. High levels of cleaved caspase- 3 confirm that cell death is due to apoptosis (35). In the present study, it was shown that in the RIPostC group, the upregulation of ALDH2 was accompanied by an elevated myocardial Bcl-2/Bax ratio and cleaved caspase-3 levels compared with those in the I/R group. The results revealed that the upregulation of the ALDH2 expression levels may contribute to reducing the occurrence of cardiomyocyte apoptosis. Although ALDH2 is essential for cell survival, the mechanism of how ALDH2 expression levels are upregulated in $\mathrm{RIPostC}$ remains to be fully elucidated.

Activation of the RISK pathway contributes to RIPreC-induced cardioprotection within the remote organ (36,37). Breivik et al (36) confirmed that RIPreC exhibited strong cardioprotective properties via the $\mathrm{PI} 3 \mathrm{~K} / \mathrm{Akt}$-dependent signaling pathway. Akt, a serine/threonie kinase, is an important mediator of the downstream effects of RISK signaling. Once Akt is activated, phosphorylated substrates are translocated from the cell membrane to various subcellular compartments. Inhibition of RISK with wortmannin reduced Akt phosphorylation and abolished ischemic pre- and postconditioning-induced protection in previous studies $(38,39)$. Lang et al (20) demonstrated that isoflurane preconditioning confers cardioprotection by activation of ALDH2 from the RISK pathway against I/R injury. The present study investigated whether the activation of ALDH2 in RIPostC also proceeded via the PI3K/Akt signaling pathway. The results demonstrated that when the rats were treated with the PI3K inhibitor wortmannin during the RIPostC intervention, the myocardial protective effect was reduced. This result further supports that RIPostC had a strong cardioprotective effect through the RISK pathway to upregulate the levels of ALDH2 expression. Furthermore, a previous study has already revealed that activation of the PI3K/Akt signaling pathway attenuates mitochondrial-mediated apoptosis. Upon activation, the endogenous protein kinase Akt confers broad cardioprotection from suppression of cell apoptosis to promotion of cellular survival in I/R injury (33). Overexpression of Akt preserves the Bcl-2 levels in mitochondria. In the present study, it was observed that RIPostC upregulated the levels of ALDH2 expression as well as inhibited the occurrence of myocardial apoptosis; however, this effect was blocked in the RIPostC+Wort group. These observations suggested that RIPostC had a cardioprotective and anti-apoptotic effect through activation of the PI3K/Akt-dependent signaling pathway to upregulate the ALDH2 expression levels. Thus, ALDH2 may have a pivotal role in cell survival and myocardial function via the PI3K/Akt-dependent signaling pathway.

In conclusion, the present study demonstrated that RIPostC protects the heart by upregulating the levels of ALDH2 against I/R-induced cardiomyocyte apoptosis, and the activation of the PI3K/Akt-dependent signaling pathway is involved in the cardioprotective effect of ALDH2.

RIPostC has a significant protective effect against myocardial ischemia and reperfusion injury in rats through activation of ALDH2. ALDH2 may be an important mediator in cardioprotection through the PI3K/Akt-dependent cell survival signaling pathway.

\section{Acknowledgements}

This study was supported by the National Natural Science Foundation of China (no. 81000074) and by the Research Fund for the Doctoral Program of Bengbu Medical College, China (no. BYkf13A05).

\section{References}

1. Yellon DM and Hausenloy DJ: Myocardial reperfusion injury. N Engl J Med 357: 1121-1135, 2007.

2. Murry CE, Jennings RB and Reimer KA: Preconditioning with ischemia: a delay of lethal cell injury in ischemic myocardium. Circulation 74: 1124-1136, 1986.

3. Kharbanda RK, Mortensen UM, White PA, et al: Transient limb ischemia induces remote ischemic preconditioning in vivo. Circulation 106: 2881-2883, 2002. 
4. Zhao ZQ, Corvera JS, Halkos ME, et al: Inhibition of myocardial injury by ischemic postconditioning during reperfusion: comparison with ischemic preconditioning. Am J Physiol Heart Circ Physiol 285: H579-H588, 2003.

5. Kerendi F, Kin H, Halkos ME, Jiang R, Zatta AJ, Zhao ZQ, Guyton RA and Vinten-Johansen J: Remote postconditioning. Brief renal ischemia and reperfusion applied before coronary artery reperfusion reduces myocardial infarct size via endogenous activation of adenosine receptors. Basic Res Cardiol 100: 404-412, 2005.

6. Gao Q, Hu J, Hu J, Yu Y, Ye H, Li Z and Guan S: Calcium activated potassium channel and protein kinase $C$ participate in the cardiac protection of remote post conditioning. Pak J Pharm Sci 26: 285-290, 2013

7. Ren C, Yan Z, Wei D, Gao X, Chen X and Zhao H: Limb remote ischemic postconditioning protects against focal ischemia in rats. Brain Res 1288: 88-94, 2009.

8. Wei M, Xin P, Li S, Tao J, Li Y, Li J, Liu M, Li J, Zhu W and Redington AN: Repeated remote ischemic postconditioning protects against adverse left ventricular remodeling and improves survival in a rat model of myocardial infarction. Circ Res 108 1220-1225, 2011.

9. Ren J: Acetaldehyde and alcoholic cardiomyopathy: lessons from the ADH and ALDH2 transgenic models. Novartis Found Symp 285: 69-79, 198-199, 2007.

10. Li SY and Ren J: Cardiac overexpression of alcohol dehydrogenase exacerbates chronic ethanol ingestion-induced myocardia dysfunction and hypertrophy: role of insulin signaling and ER stress. J Mol Cell Cardiol 44: 992-1001, 2008.

11. Hu XY, Fang Q, Wang JS, Xie JQ, Chai BS, Li FQ, Cui X and Yang Y: Over-expression of aldehyde dehydrogenase-2 protects against $\mathrm{H}_{2} \mathrm{O}_{2}$-induced oxidative damage and apoptosis in peripheral blood mononuclear cells. Acta Pharmacol Sin 32: 245-252, 2011.

12. Stewart MJ, Malek K and Crabb DW: Distribution of messenger RNAs for aldehyde dehydrogenase 1 , aldehyde dehydrogenase 2, and aldehyde dehydrogenase 5 in human tissues. J Investig Med 44: 42-46, 1996.

13. Chen CH, Budas GR, Churchill EN, Disatnik MH, Hurley TD and Mochly-Rosen D: Activation of aldehyde dehydrogenase-2 reduces ischemic damage to the heart. Science 321: 1493-1495, 2008.

14. Gao Q, Wang HJ, Wang XM, Kang PF, Yu Y, Ye HW, Zhou H and Li ZH: Activation of ALDH2 with ethanol attenuates diabetes induced myocardial injury in rats. Food Chem Toxicol 56: 419-424, 2013.

15. Fang J, Chen L, Wu L and Li W: Intra-cardiac remote ischemic post-conditioning attenuates ischemia-reperfusion injury in rats. Scand Cardiovasc J 43: 386-394, 2009.

16. Hausenloy DJ and Yellon DM: Reperfusion injury salvage kinase signalling: taking a RISK for cardioprotection. Heart Fail Rev 2: 217-234, 2007.

17. Schwartz LM and Lagranha CJ: Ischemic postconditioning during reperfusion activates Akt and ERK without protecting against lethal myocardial ischemia-reperfusion injury in pigs. Am J Physiol Heart Circ Physiol 290: H1011-H1018, 2006.

18. Dong XX, Wang YR, Qin S, Liang ZQ, Liu BH, Qin ZH and Wang Y: p53 mediates autophagy activation and mitochondria dysfunction in kainic acid-induced excitotoxicity in primary striatal neurons. Neuroscience 207: 52-64, 2012.

19. Wang YQ, Liu CH, Zhang JQ, Zhu DN and Yu BY: Protective effects and active ingredients of yi-qi-fu-mai sterile powder against myocardial oxidative damage in mice. J Pharmacol Sci 122: 17-27, 2013.

20. Lang XE, Wang X, Zhang KR, Lv JY, Jin JH and Li QS: Isoflurane preconditioning confers cardioprotection by activation of ALDH2. PLoS One 8: e52469, 2013.

21. Contractor H, Støttrup NB, Cunnington C, Manlhiot C, Diesch J, Ormerod JO, Jensen R, Bøtker HE, Redington A, Schmidt MR, Ashrafian $\mathrm{H}$ and Kharbanda RK: Aldehyde dehydrogenase-2 inhibition blocks remote preconditioning in experimental and human models. Basic Res Cardiol 108: 343, 2013.

22. Li CM, Zhang XH, Ma XJ and Luo M: Limb ischemic postconditioning protects myocardium from ischemia-reperfusion injury. Scand Cardiovasc J 40: 312-317, 2006

23. Andreka G, Vertesaljai M, Szantho G, Font G, Piroth Z, Fontos G, Juhasz ED, Szekely L, Szelid Z, Turner MS, Ashrafian H, Frenneaux MP and Andreka P: Remote ischaemic postconditioning protects the heart during acute myocardial infarction in pigs. Heart 93: 749-752, 2007.
24. Xin P, Zhu W, Li J, Ma S, Wang L, Liu M, Wei M, Redington AN: Combined local ischemic postconditioning and remote perconditioning recapitulate cardioprotective effects of local ischemic preconditioning. Am J Physiol Heart Circ Physiol 298: H1819-H1831, 2010.

25. Bøtker HE, Kharbanda R, Schmidt MR, Bøttcher M, Kaltoft AK, Terkelsen CJ, Munk K, Andersen NH, Hansen TM, Trautner S, Lassen JF, Christiansen EH, Krusell LR, Kristensen SD, Thuesen L, Nielsen SS, Rehling M, Sørensen HT, Redington AN and Nielsen TT: Remote ischaemic conditioning before hospital admission, as a complement to angioplasty, and effect on myocardial salvage in patients with acute myocardial infarction: a randomised trial. Lancet 375: 727-734, 2010.

26. Thielmann M, Kottenberg E, Boengler K, Raffelsieper C, Neuhaeuser M, Peters J, Jakob H and Heusch G: Remote ischemic preconditioning reduces myocardial injury after coronary artery bypass surgery with crystalloid cardioplegic arrest. Basic Res Cardiol 105: 657-664, 2010.

27. Loukogeorgakis SP, Williams R, Panagiotidou AT, Kolvekar SK, Donald A, Cole TJ, Yellon DM, Deanfield JE and MacAllister RJ: Transient limb ischemia induces remote preconditioning and remote postconditioning in humans by a K(ATP)-channel dependent mechanism. Circulation 116: 1386-1395, 2007.

28. Churchill EN, Disatnik MH and Mochly-Rosen D: Time-dependent and ethanol-induced cardiac protection from ischemia mediated by mitochondrial translocation of varepsilonPKC and activation of aldehyde dehydrogenase 2. J Mol Cell Cardiol 46: 278-284, 2009

29. He L, Liu B, Dai Z, Zhang HF, Zhang YS, Luo XJ, Ma QL and Peng J: Alpha lipoic acid protects heart against myocardial ischemia-reperfusion injury through a mechanism involving aldehyde dehydrogenase 2 activation. Eur J Pharmacol 678: 32-38, 2012.

30. Wang HJ, Kang PF, Wu WJ, Tang Y, Pan QQ, Ye HW, Tang B, Li ZH and Gao Q: Changes in cardiac mitochondrial aldehyde dehydrogenase 2 activity in relation to oxidative stress and inflammatory injury in diabetic rats. Mol Med Rep 8: 686-690, 2013.

31. Li ZH, Jiang CR, Xia ML, Ye HW, Guan SD and Gao Q Activation of mitochondrial aldehyde dehydrogenase 2 and inhibition of mitochondrial permeability transition pore involved in cardioprotection of ethanol postconditioning. Zhejiang Da Xue Xue Bao Yi Xue Ban 39: 566-571, 2010 (In Chinese).

32. Xu D, Guthrie JR, Mabry S, Sack TM and Truog WE: Mitochondrial aldehyde dehydrogenase attenuates hyperoxia-induced cell death through activation of ERK/MAPK and PI3K-Akt pathways in lung epithelial cells. Am J Physiol Lung Cell Mol Physiol 291: L966-L975, 2006.

33. Wang SP, Wang ZH, Peng DY, Li SM, Wang H and Wang XH: Therapeutic effect of mesenchymal stem cells in rats with intracerebral hemorrhage: reduced apoptosis and enhanced neuroprotection. Mol Med Rep 6: 848-854, 2012.

34. Sun H, Zhou F, Wang Y, Zhang Y, Chang A and Chen Q: Effects of beta-adrenoceptors overexpression on cell survival are mediated by $\mathrm{Bax} / \mathrm{Bcl}-2$ pathway in rat cardiac myocytes. Pharmacology 78: 98-104, 2006.

35. Wang YB, Qin J, Zheng XY, et al: Diallyl trisulfide induces Bcl-2 and caspase-3-dependent apoptosis via downregulation of Akt phosphorylation in human T24 bladder cancer cells. Phytomedicine 17: 363-368, 2010.

36. Breivik L, Helgeland E, Aarnes EK, Mrdalj J and Jonassen AK: Remote postconditioning by humoral factors in effluent from ischemic preconditioned rat hearts is mediated via PI3K/Akt-dependent cell-survival signaling at reperfusion. Basic Res Cardiol 106: 135-145, 2011.

37. Tamareille S, Mateus V, Ghaboura N, Jeanneteau J, Croué A, Henrion D, Furber A and Prunier F: RISK and SAFE signaling pathway interactions in remote limb ischemic perconditioning in combination with local ischemic postconditioning. Basic Res Cardiol 106: 1329-1339, 2011.

38. Mocanu MM, Bell RM and Yellon DM: PI3 kinase and not p42/p44 appears to be implicated in the protection conferred by ischemic preconditioning. J Mol Cell Cardiol 34: 661-668, 2002.

39. Skyschally A, van Caster P, Boengler K, Gres P, Musiolik J, Schilawa D, Schulz R and Heusch G: Ischemic postconditioning in pigs: no causal role for RISK activation. Circ Res 104: 15-18, 2009 . 